\title{
Acute supplementation with keto analogues and amino acids in rats during resistance exercise
}

\author{
Rosemeire Dantas de Almeida ${ }^{1,2,3}$, Eduardo Seixas Prado ${ }^{1,2,3,4}$, Carlos Daniel Llosa ${ }^{1}$, \\ Anibal Magalhães-Neto ${ }^{1,2,5}$ and Luiz-Claudio Cameron ${ }^{1,2,6 *}$ \\ ${ }^{1}$ Laboratory of Protein Biochemistry, Federal University of State of Rio de Janeiro, Av. Pasteur, 296, Urca, Rio de Janeiro, Brazil \\ ${ }^{2}$ Institute of Genetics and Biochemistry, Federal University of Uberlândia, Av. Pará, 1720, Umuarama, Uberlândia, Brazil \\ ${ }^{3}$ Biological and Health Sciences Center, Tiradentes University, Av. Murilo Dantas, S/N, Farolândia, Aracaju, Brazil \\ ${ }^{4}$ Poet João Freire Ribeiro School, Sergipe Secretary of Education, Av. Nação, S/N, Jardins, Aracaju, Brazil \\ ${ }^{5}$ Biological and Health Sciences Institute - Federal University of Mato Grosso, Rodovia MT-100, Km 3,5, Pontal do Araguaia, \\ Mato Grosso, Brazil \\ ${ }^{6}$ Graduate Program in Human Movement Science - University Castelo Branco - RJ - Brazil - Av. Salvador Allende, 6.700, \\ Recreio Rio de Janeiro, Brazil \\ (Received 17 November 2009 - Revised 11 May 2010 - Accepted 12 May 2010 - First published online 2 July 2010)
}

\begin{abstract}
During exercise, ammonia levels are related to the appearance of both central and peripheral fatigue. Therefore, controlling the increase in ammonia levels is an important strategy in ameliorating the metabolic response to exercise and in improving athletic performance. Free amino acids can be used as substrates for ATP synthesis that produces ammonia as a side product. Keto analogues act in an opposite way, being used to synthesise amino acids whilst decreasing free ammonia in the blood. Adult male rats were divided into four groups based on receiving either keto analogues associated with amino acids (KAAA) or a placebo and resistance exercise or no exercise. There was an approximately $40 \%$ increase in ammonaemia due to KAAA supplementation in resting animals. Exercise increased ammonia levels twofold with respect to the control, with a smaller increase (about $20 \%$ ) in ammonia levels due to exercise. Exercise itself causes a significant increase in blood urea levels (17\%). However, KAAA reduced blood urea levels to $75 \%$ of the pre-exercise values. Blood urate levels increased $28 \%$ in the KAAA group, independent of exercise. Supplementation increased glucose levels by $10 \%$ compared with control animals. Exercise did not change glucose levels in either the control or supplemented groups. Exercise promoted a $57 \%$ increase in lactate levels in the control group. Supplementation promoted a twofold exercise-induced increase in blood lactate levels. The present results suggest that an acute supplementation of KAAA can decrease hyperammonaemia induced by exercise.
\end{abstract}

\section{Ammonia: Urate: Urea: Resistance exercise}

Ammonia $\left(\mathrm{NH}_{3}+\mathrm{NH}_{4}{ }^{+}\right)$is a toxic metabolite with deleterious effects on the central nervous system ${ }^{(1)}$. Exercise can be used as a model to study ammonia metabolism in an intensitydependent way $^{(2-5)}$. During prolonged exercise, ammonia is mainly produced by the catabolism of amino acids ${ }^{(6)}$. On the other hand, during high-intensity exercise, the largest source of ammonia production is from AMP deamination ${ }^{(7)}$. Ammonia levels are related to the appearance of both central and peripheral fatigue ${ }^{(8)}$. Therefore, controlling increases in ammonia is an important strategy in ameliorating the metabolic response to exercise and in improving athletic performance ${ }^{(9,10)}$.

The combination of keto analogues with amino acids has been used to treat patients with chronic kidney disease (CKD), portal systemic encephalopathy and hyperammonaemia $^{(11,12)}$. Free amino acids can be used as substrates for ATP synthesis, which produces ammonia as a side product ${ }^{(13)}$.
In an opposite manner, the use of keto analogues associated with amino acids (KAAA) has been proposed as a way to synthesise amino acids whilst decreasing free ammonaemia ${ }^{(14,15)}$. During metabolism, amino acids are deaminated or transaminated to form keto acids via release of the amino group ${ }^{(16)}$. These reactions are reversible, and the use of keto analogues could reduce the blood ammonia concentration, resulting in the production of amino acids ${ }^{(17)}$. Thus, keto analogues may serve as nutritional supplements to synthesise amino acids of high biological value, especially in CKD patients. Furthermore, it is acknowledged that resistance and aerobic exercise programmes may serve important roles in the approach to the treatment, prevention and slowed progression of $\mathrm{CKD}^{(18)}$.

Although KAAA supplementation is effective in the treatment of CKD, particularly for postponing the necessity for dialysis, the use of KAAA is not popular due to its cost and

\footnotetext{
Abbreviations: CKD, chronic kidney disease; Ctl group, control group (neither keto analogues associated with amino acids nor exercise); Ex group, exercise-only group; KA group, keto analogues associated with amino acids-only group; KAAA, keto analogues associated with amino acids; KAEx group, keto analogues associated with amino acids and exercise group.
}

* Corresponding author: Professor L.-C. Cameron, email cameron@unirio.br 
the requirement for a low protein intake ${ }^{(19)}$. Thus, there has been a lack of interest in studies involving KAAA due to the lack of cost effectiveness in its use as a therapeutic agent. This lack of interest has limited the number of new papers published on the mechanism of action of KAAA. In the present study, we evaluated the effect of KAAA supplementation on ammonia production and blood urea levels during resistance exercise, showing metabolic effects that can enhance performance and post-exercise recovery.

\section{Materials and methods}

Male Wistar rats (12 weeks of age and body mass ranging from 280 to $350 \mathrm{~g}$ ) were divided into four groups of twelve animals each. The group that received only KAAA (KA group) and the group that received KAAA and exercise (KAEx group) received $0.1 \mathrm{~g} \mathrm{Ketosteril}{ }^{\circledR}$ (Fresenius Kabi, Bad Homburg, Germany) in $0.5 \mathrm{ml}$ water $(0.3 \mathrm{~g} / \mathrm{kg})$. The composition of the KAAA mixture per tablet was as follows: $\alpha$-keto analogues of isoleucine, $335 \mathrm{mg}$; leucine, $505 \mathrm{mg}$; phenylalanine, $430 \mathrm{mg}$; valine, $340 \mathrm{mg}$; $\alpha$-hydroxy analogue of methionine, $295 \mathrm{mg}$; L-lysine acetate, $75 \mathrm{mg}$; L-threonine, $265 \mathrm{mg}$; L-tryptophan, $115 \mathrm{mg}$; L-histidine, $190 \mathrm{mg}$; L-tyrosine, $150 \mathrm{mg}$. The group that received neither KAAA nor exercise (control (Ctl) group) and the group that received only exercise (Ex group) received $0.5 \mathrm{ml}$ of $0.9 \% \mathrm{NaCl}, 1 \mathrm{~h}$ before exercise by oral administration. The animals were maintained in collective cages (four per cage) at $22 \pm 2{ }^{\circ} \mathrm{C}$ with a photoperiod of $12 \mathrm{~h}$ and fed ad libitum (diet and water). The study was approved by the Ethics Committee in Research of the University of Tiradentes, and followed the Guiding Principles for Research Involving Animals and Human Beings.

Resistance exercise was performed according to a previous study $^{(20)}$ after familiarisation and determination of the load that was to be applied according to the one repetition maximum (1RM) test. Familiarisation consisted of attaching the animal to the exercise device daily without stimulating the animals to exercise, starting $6 \mathrm{~d}$ before the experiment. The 1RM test was performed $1 \mathrm{~d}$ before resistance exercise and determined the heaviest weight that could be lifted. On the day of the experiment, fifty repetitions were performed with a load equal to $75 \%$ of $1 \mathrm{RM}$. The animals were stimulated to perform the repetitions through sticker electrodes (Axelgaard ValuTrode CF3200; Axelgaard Manufacturing Co. Ltd, Fallbrook, CA, USA) placed in the tail and connected to an electrostimulator (4 mA to $15 \mathrm{~mA}$ at $1 \mathrm{~Hz}$ for $1 \mathrm{~s}$; Quark Dualpex 961; Quark Medical Products, São Paulo, Brazil).

Blood was collected through cardiac puncture before exercise ( $\mathrm{Ctl}$ and KA groups) or immediately after exercise (Ex and KAEx groups). The blood samples were immediately centrifuged to obtain sera, which was subsequently frozen and stored at $-70^{\circ} \mathrm{C}$ for future biochemical analysis. Biochemical analyses of glucose, urea, urate and creatinine concentrations were performed using commercially available spectrophotometric assays (Labtest, Minas Gerais, Brazil). Lactate and ammonia were measured using an enzymic UV method (Randox, Crumlin, Co. Antrim, UK) on a Dade Model Dimension RXL Automated Chemistry Analyzer (Dade Behring, Eschborn, Germany), and haematological parameters were analysed using a Sysmex SE-9500 Automated Hematology Analyzer (TOA Medical Electronics, Kobe, Japan). Standard curves were taken at a minimum $r$ value of 0.98 and the experimental points were always within the calibration curve and at least $20 \%$ above the lower limit of detection.

Statistical significance was evaluated by one-way ANOVA. Significances $(P<0 \cdot 05)$ were confirmed using the Tukey test as a post hoc analysis. Data are reported as mean values with their standard errors.

\section{Results}

We used a weight-lifting exercise to evaluate the effect of KAAA on blood ammonia concentration after resistance
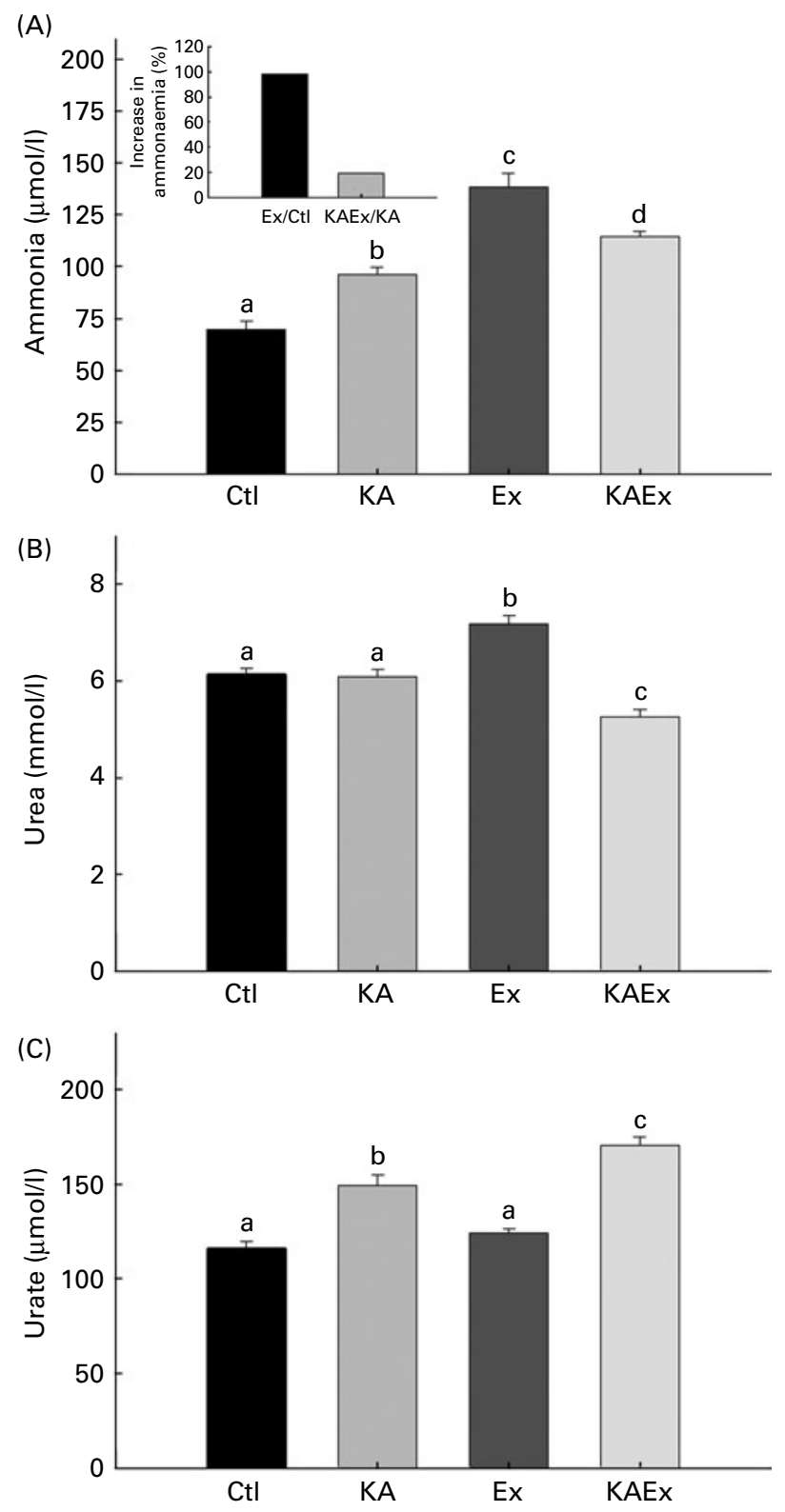

Fig. 1. Acute supplementation of keto analogues associated with amino acids (KAAA) affects ammonia (A), urea (B) and urate (C) metabolism Ctl, control group (neither KAAA nor exercise); KA, KAAA-only group; Ex, exercise-only group; KAEx, KAAA and exercise group. Values are means, with standard errors represented by vertical bars. ${ }^{a, b, c, d}$ Mean values with unlike letters were significantly different $(P<0.05)$. Inset of Fig. 1(A) shows the increase in ammonia levels in experimental and control groups when normalised against rest values. 
exercise. There was an increase of about $40 \%$ in ammonia in resting animals due to KAAA supplementation. Compared with unsupplemented controls, exercise resulted in a twofold increase in ammonia levels in the animals. However, the supplemented group had a much smaller increase (about 20\%) in ammonia levels after exercise (Fig. 1(A)). Since KAAA is proposed to decrease blood urea levels, we evaluated the response of blood urea to acute KAAA supplementation during exercise. We did not measure the effect of supplementation on blood urea at rest. With no supplementation, exercise significantly increased blood urea levels by $17 \%$ compared with the levels in the $\mathrm{Ctl}$ group. However, with KAAA supplementation, blood urea was reduced to $75 \%$ of the pre-exercise values (Fig. 1(B)).

To differentiate the ammonia production derived from AMP deamination from that derived from amino acid deamination, we measured urate, the end metabolite of inosine monophosphate. Blood urate levels increased $28 \%$ in the group supplemented with KAAA, independent of exercise. This effect was enhanced in the supplemented group after exercise. There was no change in blood urate levels in the control group in response to exercise (Fig. 1(C)).

KAAA supplementation has been shown to increase creatinine clearance. Our study model did not detect a change in blood creatinine in response to exercise. However, blood creatinine decreased by $40 \%$ in the groups supplemented with KAAA, independent of exercise (Table 1).

To understand the effect of KAAA on glucose maintenance, we measured glucose levels after exercise. Supplementation increased glucose levels in resting animals by $10 \%$. Exercise did not change glucose levels in either the $\mathrm{Ctl}$ or KAEx groups (Table 1).

Blood lactate is an indicator of glucose utilisation during exercise. Exercise promoted a $57 \%$ increase in blood lactate in the $\mathrm{Ctl}$ group. The supplementation promoted a twofold exercise-induced increase in blood lactate (Table 1).

\section{Discussion}

It is widely reported that ammonia production increases during exercise and that ammonia could be a deleterious metabolite that promotes fatigue ${ }^{(8,20,21)}$. The production of ammonia can lead to significantly elevated systemic ammonia levels to levels between 90 and $>200 \mu \mathrm{mol} / \mathrm{l}$. Patients with either liver or kidney disease also show sharp increases in ammonia levels that may range from 70 to $300 \mu \mathrm{mol} / 1$ in liver disorder patients $^{(1,22)}$. Patients with CKD have lower peak levels of ammonia during exercise, but experience ammonia increases of about 30-60\% compared with resting values ${ }^{(1,23,24)}$. KAAA has been widely used as a supplement to treat patients with kidney failure and as a therapeutic agent for liver failure and encephalopathy ${ }^{(12)}$. Additionally, regular physical activity and close clinical and dietary monitoring, including the use of keto analogues, should be recommended in patients with $\mathrm{CKD}^{(25)}$.

One of the problems associated with human studies has been ensuring that subjects have adhered to the recommended diet and have properly taken the supplements. Here, we used a previously described resistance exercise animal method ${ }^{(19)}$ to investigate a possible ammonia-chelating effect of KAAA during exercise in rats. The production of ammonia during exercise occurs via both AMP deamination and branchedchain amino acid metabolism ${ }^{(1)}$.

The use of KAAA increased ammonaemia during the resting state, demonstrating that amino acid metabolism during exercise is associated with anaplerosis of Krebs cycle intermediates $^{(26,27)}$. Increases in ammonia levels in response to exercise can be managed through the use of amino acids or carbohydrates that interfere with ammonia metabolism ${ }^{(28)}$. It is possible to propose that the amino acids in the supplement are being using either as carbon skeleton donors to obtain energy or as gluconeogenic precursors. Even with an increase in ammonia levels at rest, KAAA supplementation was able to reduce the exercise-induced increase in blood ammonia by $80 \%$. When compared with the non-supplemented exercise group, the absolute decrease was $20 \%$. Previous data in our laboratory showed that there is a habituation of basal ammonia levels in response to amino acid supplementation, since the resting ammonia level decreases with an increase in basal blood urea levels correlated to supplementation time ${ }^{(3)}$. On the basis of these data, we postulate that the effect of KAAA supplementation on basal ammonia levels can be diminished by chronic KAAA use.

Our exercise model increased ammonia and urea levels in animals without any changes in urate levels. It has been pointed out that excess ammonia is metabolised to urea by the liver for excretion to minimise toxicity ${ }^{(2)}$. During exercise, KAAA was able to decrease the blood urea concentration to $75 \%$ of the resting urea level. This finding is related to the widely described therapeutic effect of KAAA (for a review, see Savica et al. $\left.{ }^{(13)}\right)$. Urate appears more quickly in blood in response to exercise compared with urea ${ }^{(29)}$. KAAA supplementation increased resting urate levels. However, we detected changes in blood urate in the supplemented exercise group when compared with the non-supplemented exercise group after resistance exercise. It is known that during

Table 1. Creatinine, glucose and lactate as obtained from the four supplementation protocols (Mean values with their standard errors)

\begin{tabular}{|c|c|c|c|c|c|c|c|c|}
\hline \multirow{2}{*}{$\begin{array}{l}\text { Group... } \\
\text { Parameter }\end{array}$} & \multicolumn{2}{|c|}{ Ctl } & \multicolumn{2}{|c|}{$\mathrm{KA}$} & \multicolumn{2}{|c|}{ Ex } & \multicolumn{2}{|c|}{ KAEx } \\
\hline & Mean & SE & Mean & SE & Mean & SE & Mean & SE \\
\hline ne $(\mathrm{mmol} / \mathrm{l})$ & $47 \cdot 66^{a}$ & 0.91 & $28 \cdot 89^{b}$ & 0.71 & $47 \cdot 49^{a}$ & 0.76 & $27 \cdot 76^{\mathrm{b}}$ & 0.76 \\
\hline Glucose $(\mathrm{mmol} / \mathrm{l})$ & $6 \cdot 11^{\mathrm{a}}$ & 0.12 & $6 \cdot 64^{b}$ & 0.20 & $6.06^{\mathrm{a}}$ & 0.12 & $6 \cdot 81^{\mathrm{b}}$ & 0.16 \\
\hline Lactate $(\mathrm{mmol} / \mathrm{l})$ & $2 \cdot 66^{\mathrm{a}}$ & 0.08 & $2 \cdot 67^{\mathrm{a}}$ & 0.07 & $4 \cdot 18^{\mathrm{b}}$ & 0.09 & $5 \cdot 44^{c}$ & 0.09 \\
\hline
\end{tabular}

$\mathrm{Ctl}$, control (no keto analogues associated with amino acids or exercise); KA, keto analogues associated with amino acids only; Ex, exercise only; KAEx, keto analogues associated with amino acids and exercise.

a,b,c Mean values within a row with unlike superscript letters were significantly different $(P<0.05$; ANOVA). 
high-intensity exercise such as resistance exercise, the largest quantity of urate is produced when the ATP:ADP ratio decreases which leads to increases in both AMP deamination and urate synthesis ${ }^{(8)}$. It is possible that our resistance exercise model with fifty repetitions activates pathways associated with resistance and prolonged exercise. KAAA supplementation promoted an increase in creatinine clearance. This is a welldescribed effect of this supplement in chronically ill patients $^{(12)}$. Taking these results together, we postulate that the majority of ammonia production results from the deamination of amino acids instead of $\operatorname{AMP}^{(7,8,30)}$.

Some studies have shown that amino acid supplementation increased the pool of Krebs cycle intermediates during exercise $^{(5,31)}$. KAAA supplementation produced a $10 \%$ increase in resting glucose levels that were maintained even after exercise. Since KAAA is a mixture of ketogenic and glucogenic keto analogues and amino acids, we postulate that KAAA provide glucose for exercise. It is important to state that the use of the amino acids from KAAA as carbon skeleton donors augments the net ammonia release. On the other hand, the anaplerosis using the keto analogues does not increase ammonia release. However, both situations increase ATP synthesis, leading to a decelerating ammonia production due to AMP deamination.

The results of the present study showed that KAAA supplementation exacerbated blood lactate levels after exercise. It is known that lactate is formed during glycolysis in active skeletal muscles and many conditions can attenuate lactate levels during exercise, such as muscle glycogen depletion ${ }^{(32)}$. Thus, such alterations in the present study may be explained by KAAA providing glucose for exercise through gluconeogenesis. Since the central nervous system has no effective urea cycle and depends on the synthesis of glutamine for removal of the excess ammonia ${ }^{(9,33)}$, high levels of blood ammonia have been proposed to be related to the development of both local and central fatigue ${ }^{(9,21,34)}$. Here, we describe for the first time that acute supplementation of KAAA can be used to reduce the increase in ammonia levels caused by resistance exercise. The practical significance of these findings may be important for the individual exerciser and merits further research to examine the efficacy of chronic KAAA intake. Therefore, we believe that the present study contributes important data to our understanding of metabolism and that these findings could be helpful for the development of future therapies.

\section{Acknowledgements}

The present study was supported in part by Tiradentes University.

R. D. A., E. S. P. and L.-C. C. were responsible for the study design. All authors contributed to data collection and interpretation, and manuscript writing.

The authors have no conflicts of interest to declare.

\section{References}

1. Wilkinson DJ, Smeeton NJ \& Watt PW (2010) Ammonia metabolism, the brain and fatigue; revisiting the link. Prog Neurobiol 91, 200-219.

2. Felipo V \& Butterworth RF (2002) Neurobiology of ammonia. Prog Neurobiol 67, 259-279.
3. Bassini-Cameron A, Monteiro AN, Gomes A, et al. (2008) Glutamine protects against increases in blood ammonia in football players in an exercise intensity-dependent way. $\mathrm{Br} \mathrm{J}$ Sports Med 42, 260-266.

4. Alvear-Ordenes I, García-López, De Paz JA, et al. (2005) Sweat lactate, ammonia, and urea in rugby players. Int J Sports Med 26, 632-637.

5. Roeykens J, Magnus L, Rogers R, et al. (1998) Blood ammonia-heart rate relationship during graded exercise is not influenced by glycogen depletion. Int J Sports Med 19, 26-31.

6. Snow R, Carey M, Stathis C, et al. (2000) Effect of carbohydrate ingestion on ammonia metabolism during exercise in humans. J Appl Physiol 88, 1576-1580.

7. Graham TE \& MacLean DA (1992) Ammonia and amino acid metabolism in human skeletal muscle during exercise. Can $J$ Physiol Pharmacol 70, 132-141.

8. Hellsten Y, Richter EA, Kiens B, et al. (1999) AMP deamination and purine exchange in human skeletal muscle during and after intense exercise. J Physiol 520, 909-920.

9. Nybo L, Dalsgaard MK, Steensberg A, et al. (2005) Cerebral ammonia uptake and accumulation during prolonged exercise in humans. J Physiol 15, 285-290.

10. Hirai T, Minatogawa Y, Hassan AM, et al. (1995) Metabolic interorgan relations by exercise of fed rats: carbohydrates, ketone body, and nitrogen compounds in splanchnic vessels. Physiol Behav 57, 515-522.

11. Meneguello MO, Mendonça JR, Lancha AH Jr, et al. (2003) Effect of arginine, ornithine and citrulline supplementation upon performance and metabolism of trained rats. Cell Biochem Funct 21, 85-91.

12. Walser M (1990) Role of branched-chain ketoacids in protein metabolism. Kidney Int 38, 595-604.

13. Savica V, Santoro D, Ciolino F, et al. (2005) Nutritional therapy in chronic kidney disease. Nutr Clin Care 8, 70-76.

14. Wu G (2009) Amino acids: metabolism, functions and nutrition. Amino Acids 37, 1-17.

15. Walser M (1975) Ketoacids in the treatment of uremia. Clin Nephrol 3, 180-186.

16. Furst $\mathrm{P}$ (1989) Amino acid metabolism in uremia. J Am Coll Nutr 8, 310-323.

17. Walser M (1975) Nutritional effects of nitrogen-free analogues of essential amino acids. Life Sci 17, 1011-1020.

18. Johansen KL (2005) Exercise and chronic kidney disease: current recommendations. Sports Med 35, 485-499.

19. Burns J, Cresswell E, Ell S, et al. (1978) Comparison of the effects of keto acid analogues and essential acids on nitrogen homeostasis in uremic patients on moderately protein restricted diets. Am J Clin Nutr 31, 1767-1775.

20. Tamaki T, Shuichi U \& Shoichi N (1992) A weight-lifiting exercise model for inducing hypertrophy in the hindlimb muscles of rats. Med Sci Sports Exerc 24, 881-886.

21. Banister EW \& Cameron BJ (1990) Exercise-induced hyperammonemia: peripheral and central effects. Int J Sports Med 11, $129-142$.

22. Olde Damink SW, Deutz NE, Dejong CH, et al. (2002) Interorgan ammonia metabolism in liver failure. Neurochem Int $\mathbf{4 1}$ $177-188$

23. Pimentel JL Jr, Brusilow SW \& Mitch WE (1994) Unexpected encephalopathy in chronic renal failure: hyperammonemia complicating acute peritonitis. $J$ Am Soc Nephrol 5, 1066-1073.

24. Deferrari G, Garibotto G, Robaudo C, et al. (1981) Brain metabolism of amino acids and ammonia in patients with chronic renal insufficiency. Kidney Int 20, 505-510.

25. Cupisti A, Licitra R, Chisari C, et al. (2004) Skeletal muscle and nutritional assessment in chronic renal failure patients on a protein-restricted diet. J Intern Med 255, 115-124. 
26. Gibala MJ, Lozej M, Tarnopolsky MA, et al. (1997) Low glycogen and branched-chain amino acid ingestion do not impar anaplerosis during exercise in humans. Am J Physiol 272, E239-E244.

27. Gibala MJ, MacLean C, Graham TE, et al. (1998) Tricarboxylic acid cycle intermediate pool size and estimated cycle flux in human muscle during exercise. Am $J$ Physiol 275, E235-E242.

28. Carvalho-Peixoto J, Alves RC \& Cameron LC (2007) Glutamine and carbohydrate supplements reduce ammonemia increase during endurance field exercise. Appl Physiol Nutr Metab 32, 1186-1190.

29. Bessa A, Nissenbaum M, Monteiro A, et al. (2008) Highintensity ultraendurance promotes early release of muscle injury markers. Br J Sports Med 42, 589-593.
30. Zhao F, Xu S, Du L, et al. (2000) AMP makes native snake muscle fructose-1,6-bisphosphatase to an alkaline enzyme. Sci China C Life Sci 43, 1-7.

31. Bruce M, Constantini-Teodosiiou D, Greenhaff PL, et al. (2001) Glutamine supplementation promotes anaplerosis but not oxidative energy delivery in human skeletal muscle. Am $J$ Physiol 280, E669-E675.

32. Mourtzakis M \& Graham TE (2002) Glutamate ingestion and its effects at rest and during exercise in humans. J Appl Physiol 93, $1251-1259$.

33. Suárez I, Bodega G \& Fernandez B (2002) Glutamine synthase in brain: effect of ammonia. Neurochem Int 41, 123-142.

34. Cooper AJ (2001) Role of glutamine in cerebral nitrogen metabolism and ammonia neurotoxicity. Ment Retard Dev Disabil Res Rev 7, 280-286. 